\title{
Supernova deleptonization asymmetry: Impact on self-induced flavor conversion
}

\author{
Sovan Chakraborty, ${ }^{1}$ Georg Raffelt, ${ }^{1}$ Hans-Thomas Janka, ${ }^{2}$ and Bernhard Müller ${ }^{3}$ \\ ${ }^{1}$ Max-Planck-Institut für Physik (Werner-Heisenberg-Institut), Föhringer Ring 6, 80805 München, Germany \\ ${ }^{2}$ Max-Planck-Institut für Astrophysik, Karl-Schwarzschild-Str. 1, 85748 Garching, Germany \\ ${ }^{3}$ Monash Centre for Astrophysics, School of Physics and Astronomy, \\ Building 79P, Monash University, Victoria 3800, Australia
}

(Dated: December 3, 2014, revised September 4, 2015)

\begin{abstract}
During the accretion phase of a core-collapse supernova (SN), the deleptonization flux has recently been found to develop a global dipole pattern (LESA - Lepton Emission Self-sustained Asymmetry). The $\nu_{e}$ number flux $F_{\nu_{e}}$ is much larger than $F_{\bar{\nu}_{e}}$ in one direction, whereas they are approximately equal, or even $F_{\nu_{e}} \lesssim F_{\bar{\nu}_{e}}$, in the opposite direction. We use a linearized stability analysis in a simplified SN model to study the impact of the $\nu_{e}-\bar{\nu}_{e}$ flux asymmetry on self-induced neutrino flavor conversion. While a small lepton-number flux facilitates self-induced flavor conversion, "multiangle matter suppression" is more effective. Overall we find that for large matter densities which are relevant below the shock wave, self-induced flavor conversion remains suppressed in the LESA context and thus irrelevant for neutrino-driven explosion dynamics.
\end{abstract}

PACS numbers: 14.60.Pq, 97.60.Bw

\section{INTRODUCTION}

The neutrino and antineutrino flux spectra emitted by a core-collapse supernova (SN) significantly depend on flavor. Therefore, flavor conversion can strongly modify what neutrinos do after decoupling, notably in driving the explosion, determining nucleosynthesis yields in the neutrino-driven wind, and the expected signal in largescale detectors [1. Even though neutrino mixing angles are large, in a dense medium the eigenstates of propagation and those of interaction are very nearly the same [2]. Therefore, significant flavor conversion would only occur by the MSW effect 3, 4, at a large distance from the collapsed core.

This situation can fundamentally change when taking neutrino-neutrino refraction into account [5]. It can lead to self-induced flavor conversion [6, 7] even when the matter density is large and therefore the effective mixing angle is small 8 10. The propagation eigenmodes of the collective neutrino ensemble include run-away solutions in flavor space [11, leading to this phenomenon. One question is if self-induced flavor conversion would occur in regions below the stalled shock wave during the SN accretion phase. Flavor conversion could then modify neutrino energy deposition and impact the explosion dynamics in the framework of the neutrino-driven mechanism of SN explosion.

While self-induced flavor conversion can occur at much higher density than MSW conversion, it is still suppressed by the "multi-angle matter effect" [12, although the exact conditions where conversions would occur require a linearized stability analysis or a numerical solution of the neutrino-flavor equations of motion. Dedicated studies, using one-dimensional (1D) SN models found that the "onset radius" of self-induced flavor conversion would always lie beyond the shock front where matter densities are much smaller [13, 14]. Subsequent studies using other SN models found similar results [15, 16].
Since that time, our theoretical understanding of both self-induced flavor conversion and of flavor-dependent SN neutrino emission have both evolved. We are here especially concerned with developments having to do with spontaneous breaking of symmetries that were previously taken for granted. Axial symmetry of the neutrino radiation field around a given radial direction had been assumed in studies of collective flavor oscillations. However, a new class of run-away solutions breaks this symmetry spontaneously, allowing self-induced flavor conversion for both neutrino mass orderings $[17-22]$. Moreover, spatial uniformity can also be broken spontaneously, leading to small-scale instabilities $[23-26]$. However, very recently it was shown that in the SN context, after including multi-angle matter effects, the uniform mode provides the most sensitive test for instability [27]. Therefore, in our present study we do not worry about small-scale instabilities when addressing the impact of an unusually small lepton-number flux.

The other new finding is that SN neutrino emission in 3D models can develop a global dipole pattern termed LESA for Lepton Emission Self-sustained Asymmetry [28, 29]. While the overall neutrino luminosity remains nearly spherically symmetric, the deleptonization flux $\left(\nu_{e}\right.$ minus $\bar{\nu}_{e}$ ) develops a strong dipole pattern within the first $\sim 150 \mathrm{~ms}$ after collapse irrespective of other hydrodynamical instabilities, notably even in the presence of the standing accretion shock instability (SASI). More recent 3D models of the Garching group [30, 31] as well as the Oak Ridge group [32] show explosions. While all Garching models exhibit the LESA effect very clearly and suggest that it persists at least until some $100 \mathrm{~ms}$ after the onset of the explosion 33, the 3D explosion model of Oak Ridge shows a less stationary dipole with an amplitude of just about $10 \%$ of the monopole over most of the pre-explosion evolution and a more LESA-like episode with a dipole-to-monopole ratio of $\sim 25 \%$ and stable direction only in a time interval between 360 and $410 \mathrm{~ms}$ 
after bounce [34. The reason for these differences between the Garching and Oak Ridge models is not clear yet, but they may be connected to different structural or transport conditions in the newly formed neutron star. The LESA phenomenon, when it is fully developed, implies that during the accretion phase, the deleptonization flux emerges primarily in one hemisphere. In some directions of the opposite hemisphere, it can be very small and sometimes even slightly negative.

One may wonder if an unusually small lepton-number flux would facilitate self-induced flavor conversion, perhaps even below the shock front, and thus would require a fundamental re-examination of neutrino heating in directions of small lepton-number flux. For isotropic neutrinos or SN neutrinos in the single-angle approximation, selfinduced flavor conversion is unavoidable in a $\nu_{e}-\bar{\nu}_{e}$ symmetric ensemble 36 39]. On the other hand, multi-angle effects caused by matter or even by neutrinos themselves can suppress the instability. The main purpose of our work is a parametric study of the impact of the $\nu_{e}-\bar{\nu}_{e}$ flux asymmetry on self-induced flavor conversion.

We use a linearized stability analysis, where we neglect several issues that have been discussed in the recent literature. We ignore spin-flip effects caused by neutrino magnetic dipole moments [40 42] or simply by refraction in inhomogeneous or anisotropic media 43 46, and we ignore the role of anomalous neutrino-antineutrino pair correlations [47 50]. Realistically, all of these are probably small effects. It could be more questionable that we also ignore the "halo flux" produced by residual scattering beyond the neutrino sphere and especially its "backward going" component [51 53]. Moreover, we do not worry about the question if our assumed quasi-stationary neutrino source indeed produces a quasi-stationary solution. A true assessment of these latter assumptions requires a deeper conceptual development irrespective of the LESA context.

We begin in Sec. II with setting up the linear stability analysis and clarifying our conventions and normalizations of crucial input parameters. In Sec. [II] we perform the stability analysis for a simplified setup and we discuss the role of the $\nu_{e}-\bar{\nu}_{e}$ asymmetry. We conclude in Sec. IV with a summary and discussion of our findings.

\section{FLAVOR STABILITY CONDITIONS}

\section{A. Equations of motion}

We set up our stability analysis along the lines of Ref. [17, slightly adapting some conventions. The flavordependent SN neutrino fluxes depend, at a given radius $r$, on energy $E$. The neutrino direction of propagation in the transverse direction is described by the zenith angle $\theta$ relative to the radial direction and by the azimuth angle $\varphi$. We assume that the fluxes do not depend on coordinates in the transverse direction. The impact of smallscale variations in the transverse direction was recently studied in Ref. 27] with the result that, for a stability analysis, the largest-scale modes are most relevant. We use a very basic "neutrino bulb model" to describe the initial fluxes, i.e., we assume that neutrinos are produced at a spherical surface with radius $R$ and emitted isotropically (blackbody like) into the outside half-space. It is convenient to describe the zenith direction with a fixed label $u=\sin ^{2} \theta_{R}$, where $\theta_{R}$ is the zenith angle at radius $R$. Our blackbody like emission model corresponds to a uniform distribution on the interval $0 \leq u \leq 1$.

We stress that our reference radius $R$ is not a physical quantity but a quantity of mathematical convenience. It need not coincide with the neutrino sphere $R_{\nu}$ where typical neutrinos decouple and which is the physical emission region. Neutrinos emerging at $R_{\nu}$ tend to show a forward-peaked zenith-angle distribution. The approximate width of this distribution provides us with the approximate radius $R$ of a hypothetical blackbody surface that would produce a flux with similar angular divergence. Our main point of convenience is to use a $u$ distribution on the unit interval and we define $R$ accordingly.

Notice that we do not integrate the flavor evolution equations, but the philosophy of the stability analysis is to assume that nothing happens until our chosen test radius $r$, i.e., neutrinos have remained in flavor eigenstates which coincide with propagation eigenstates in a dense medium. Therefore, the exact path taken before reaching the radius $r$ is irrelevant. We only need to know the flavor-dependent number fluxes and their angular divergence at radius $r$.

We combine the flavor-dependent fluxes, assumed to be stationary, into a matrix $\mathrm{F}_{r, E, u, \varphi}$ which depends on the variables denoted by subscripts. Matrices in flavor space are written as capital sans-serif letters. The diagonal entries are the $4 \pi$ equivalent ordinary neutrino fluxes $F_{\alpha}$ for species $\alpha$. Notice that $F_{\alpha}$ is differential with regard to energy $E$, i.e., it is a flux spectrum, and it is also differential with regard to the zenith-angle variable $u$ and the azimuth angle $\varphi$. The off-diagonal elements of $F$ contain flavor coherence information.

We use negative energies $E$ and negative flux values $F_{\alpha}$ to denote antineutrinos. This "flavor isospin convention" considerably simplifies the analysis because we do not need to distinguish explicitly between neutrinos and antineutrinos in the equations. However, this notation can also be confusing. $F_{\nu_{e}}(E)$ is a positive number for a positive $E$, but a negative number for negative $E$ and then denotes the antineutrino flux in the sense that $F_{\bar{\nu}_{e}}(|E|)=-F_{\nu_{e}}(-|E|)$ if we take $F_{\bar{\nu}_{e}}(|E|)$ to be the usual positive-valued $\bar{\nu}_{e}$ flux.

Assuming that the solution does not depend on global direction, the radial evolution is given by [11, 17]

$$
\mathrm{i} \partial_{r} \mathrm{~F}_{E, u, \varphi}=\left[\mathrm{H}_{E, u, \varphi}, \mathrm{F}_{E, u, \varphi}\right]
$$

where we have suppressed the index $r$ on all quantities. 
The Hamiltonian matrix governing the evolution is 17

$$
\begin{aligned}
\mathrm{H}_{E, u, \varphi} & =\frac{1}{v_{u}}\left(\frac{\mathrm{M}^{2}}{2 E}+\sqrt{2} G_{\mathrm{F}} \mathrm{N}_{\ell}\right) \\
& +\frac{\sqrt{2} G_{\mathrm{F}}}{4 \pi r^{2}} \int d \Gamma^{\prime}\left(\frac{1-v_{u} v_{u^{\prime}}-\boldsymbol{\beta}_{u} \cdot \boldsymbol{\beta}_{u^{\prime}}}{v_{u} v_{u^{\prime}}}\right) \mathrm{F}^{\prime},
\end{aligned}
$$

where $\mathrm{M}^{2}$ is the neutrino mass-squared matrix, causing vacuum oscillations. The factor $v_{u}^{-1}$ arises from projecting the propagation path onto the radial direction. This factor causes the multi-angle matter effect: neutrinos traveling in different directions accrue different phases along the radial direction. The matter term is given by the matrix $\mathrm{N}_{\ell}$ of net charged-lepton densities which is diagonal in the weak-interaction basis. The third term represents neutrino-neutrino refraction which is given by the phase-space integral $\int d \Gamma^{\prime}=\int_{-\infty}^{+\infty} d E^{\prime} \int_{0}^{1} d u^{\prime} \int_{0}^{2 \pi} d \varphi^{\prime}$, where $\mathrm{F}^{\prime}$ is understood as $\mathrm{F}_{E^{\prime}, u^{\prime}, \varphi^{\prime}}$ at radius $r$. The radial velocity of a given mode is $v_{u}=\left(1-u R^{2} / r^{2}\right)^{1 / 2}$ [39. The transverse velocity $\boldsymbol{\beta}$ depends on the azimuth angle $\varphi$. One finds $\left|\boldsymbol{\beta}_{u}\right|=\sqrt{u} R / r$ and $\boldsymbol{\beta}_{u} \cdot \boldsymbol{\beta}_{u^{\prime}}=$ $\sqrt{u u^{\prime}}(R / r)^{2} \cos \left(\varphi-\varphi^{\prime}\right)$ [17.

We restrict our stability analysis to a two-flavor system and express the flux matrices in the form

$$
\mathrm{F}=\frac{\operatorname{Tr} \mathrm{F}}{2}+\frac{F_{\nu_{e}}^{R}-F_{\nu_{x}}^{R}}{2} \mathrm{~S}, \quad \mathrm{~S}=\left(\begin{array}{cc}
s & S \\
S^{*} & -s
\end{array}\right),
$$

where $s$ is real, $S$ complex, and $s^{2}+|S|^{2}=1$. The fluxes with superscript $R$ are the ones at radius $R$ where $s=1$ and $S=0$. Since the overall neutrino flux is conserved, the trace term is conserved and drops out of the commutator equation. Because the $F_{\alpha}$ are $4 \pi$ equivalent fluxes, they do not decrease with distance, only the flavor content evolves. The flavor evolution is encoded in the normalized matrix $\mathrm{S}$.

Next we change the energy variable $E$ to the frequency variable $\omega=\Delta m^{2} / 2 E$ which is more convenient in the flavor oscillation context. Negative $\omega$ values denote antineutrino modes of the neutrino field. We imagine that self-induced flavor conversion is driven by the atmospheric mass difference. Our sign convention is such that a positive $\Delta m^{2}$ refers to inverted mass ordering. Actually we will always assume $\Delta m^{2}$ to be a positive parameter and will explain later on how to implement normal mass ordering in our equations.

As far as flavor conversion is concerned, what enters is the two-flavor flux difference $F_{\nu_{e}}-F_{\nu_{x}}$, not the individual flavor fluxes. Therefore, it proves useful to introduce a dimensionless neutrino spectrum in the form

$$
g=\frac{F_{\nu_{e}}^{R}-F_{\nu_{x}}^{R}}{\frac{1}{2} \int d \Gamma\left|F_{\nu_{e}}^{R}-F_{\nu_{x}}^{R}\right|},
$$

where $g$ and all fluxes depend on $\omega, u$, and $\varphi$, and

$$
\int d \Gamma=\int_{-\infty}^{+\infty} d \omega \int_{0}^{1} d u \int_{0}^{2 \pi} d \varphi
$$

We recall that antineutrino fluxes in the flavor-isospin convention are negative and that typically the $\nu_{e}$ flux is larger than the $\nu_{x}$ flux, and similar for antineutrinos. Therefore, in the SN context, $g$ is typically positive for positive $\omega$ (neutrinos) and negative for negative $\omega$ (antineutrinos), although the opposite sign can appear for some range of $\omega$, corresponding to a "crossed over" number flux between $\nu_{e}$ and $\nu_{x}$.

Our normalization differs from our previous studies where we often used $\int_{-\infty}^{0} d \omega \int_{0}^{1} d u \int_{0}^{2 \pi} d \varphi\left|F_{\bar{\nu}_{e}}^{R}-F_{\bar{\nu}_{x}}^{R}\right|$ in the denominator, i.e., the total number flux of $\bar{\nu}_{e}$ minus that of $\bar{\nu}_{x}$. However, in the maximal lepton-flux direction of our $3 \mathrm{D}$ models, this quantity becomes very small or even vanishes and thus can not be used to normalize other fluxes. Moreover, our new definition is more symmetric and more intuitive.

With the spectrum thus normalized, we define the $\nu_{e}-\bar{\nu}_{e}$ asymmetry parameter as

$$
\epsilon=\int d \Gamma g(\omega, u, \varphi)=\frac{\int d \Gamma\left(F_{\nu_{e}}^{R}-F_{\nu_{x}}^{R}\right)}{\frac{1}{2} \int d \Gamma\left|F_{\nu_{e}}^{R}-F_{\nu_{x}}^{R}\right|} .
$$

If we denote with $N_{\alpha}$ the total positive number flux for neutrinos or antineutrinos $\alpha$, the asymmetry parameter corresponds to

$$
\epsilon=2 \frac{\left(N_{\nu_{e}}-N_{\bar{\nu}_{e}}\right)-\left(N_{\nu_{x}}-N_{\bar{\nu}_{x}}\right)}{\left(N_{\nu_{e}}+N_{\bar{\nu}_{e}}\right)-\left(N_{\nu_{x}}+N_{\bar{\nu}_{x}}\right)} .
$$

In the SN context we have approximately $N_{\nu_{x}}=N_{\bar{\nu}_{x}}$, further simplifying this expression. The normalization in our previous papers was $\epsilon=\left(N_{\nu_{e}}-N_{\bar{\nu}_{e}}\right) /\left(N_{\bar{\nu}_{e}}-N_{\bar{\nu}_{x}}\right)$ in this notation.

The main point of our study is to investigate how flavor stability depends on the asymmetry parameter $\epsilon$. In the LESA context, we have a strong lepton-number flux dipole, whereas the $\nu_{e}$ plus $\bar{\nu}_{e}$ and the $\nu_{x}$ plus $\bar{\nu}_{x}$ fluxes remain nearly $4 \pi$ symmetric, i.e., the denominator in our definition of $\epsilon$ is nearly independent of direction.

\section{B. Linearization}

We finally linearize the equations of motion in two ways. We assume $|S| \ll 1$ as we want to investigate exponentially growing modes in $S$, implying $s=1$ to linear order. Furthermore, we use the large-distance approximation, $r \gg R$, leading to [17,

$$
\begin{aligned}
\mathrm{i} \partial_{r} S & =[\omega+u(\lambda+\epsilon \mu)] S \\
& -\mu \int d \Gamma^{\prime}\left[u+u^{\prime}-2 \sqrt{u u^{\prime}} \cos \left(\varphi-\varphi^{\prime}\right)\right] g^{\prime} S^{\prime},
\end{aligned}
$$

where $S$ depends on $\omega, u$ and $\varphi$, whereas $S^{\prime}$ and $g^{\prime}$ depend on $\omega^{\prime}, u^{\prime}$ and $\varphi^{\prime}$. We then seek eigenvalues $\Omega=\gamma+i \kappa$ for solutions of the form $S(r, \omega, u, \varphi)=Q_{\omega, u, \varphi} e^{-i \Omega r}$, where a positive imaginary part $\kappa$ reflects unstable solutions.

The effective multi-angle strength of the neutrinoneutrino and neutrino-matter interaction are described 
by the parameters

$$
\begin{aligned}
\mu & =\sqrt{2} G_{\mathrm{F}} \frac{\left(N_{\nu_{e}}+N_{\bar{\nu}_{e}}\right)-\left(N_{\nu_{x}}+N_{\bar{\nu}_{x}}\right)}{8 \pi r^{2}} \frac{R^{2}}{2 r^{2}}, \\
\lambda & =\sqrt{2} G_{\mathrm{F}} n_{e} \frac{R^{2}}{2 r^{2}},
\end{aligned}
$$

where $n_{e}$ is the net electron density $\left(e^{-}\right.$minus $\left.e^{+}\right)$. On the other hand, $n_{\nu_{e}}=N_{\nu_{e}} /\left(4 \pi r^{2}\right)$ is the $\nu_{e}$ density at radius $r$ and $n_{\bar{\nu}_{e}}=N_{\bar{\nu}_{e}} /\left(4 \pi r^{2}\right)$ is the $\bar{\nu}_{e}$ number density. Therefore, in the definition of $\mu$, besides subtracting the $\nu_{x}$ and $\bar{\nu}_{x}$ densities, we use the sum $\left(n_{\nu_{e}}+n_{\bar{\nu}_{e}}\right) / 2$ to describe the neutrino matter effect. Our definition of $\mu$ differs somewhat from the previous literature, corresponding to our modified definition of $g$. What enters in the equations is the combination $\mu g_{\omega, u, \varphi}$, which is independent of chosen normalizations of either quantity.

In the LESA context, the total number fluxes $N_{\nu_{e}}+$ $N_{\bar{\nu}_{e}}$ and $N_{\nu_{x}}+N_{\bar{\nu}_{x}}$ are almost $4 \pi$ symmetric. Therefore, our new normalization of $\mu$ implies that it is nearly $4 \pi$ symmetric in our models.

Notice also that $\mu$ explicitly shows the usual $r^{-4}$ variation with distance. Therefore, we will write

$$
\mu=\mu_{R}(R / r)^{4} .
$$

In our explicit analysis we will use $\mu_{R}=2 \times 10^{5} \mathrm{~km}^{-1}$ and $R=15 \mathrm{~km}$, whereas the physical neutrino sphere is approximately at $R_{\nu}=30 \mathrm{~km}$. Either $\mu$ or $r$ can be used as a measure of distance from the $\mathrm{SN}$ core.

\section{Axially symmetric neutrino emission}

We assume axially symmetric neutrino emission, implying $g(\omega, u, \varphi) \rightarrow g(\omega, u) / 2 \pi$, but not necessarily an axially symmetric solution. In the LESA context, the local neutrino radiation field at some distance $r$ and some specific direction is not axially symmetric. However, if we assume LESA to represent an exact dipole, the system shows global axial symmetry relative to the dipole direction. Our schematic neutrino distributions always refer to the direction of minimal or maximal lepton-number emission, i.e., to the positive or negative LESA dipole directions. In these extreme lepton-asymmetry directions the fluxes can be assumed to be locally axially symmetric and in this sense axial symmetry pertains to the emitted fluxes. We do not think that our overall conclusions are strongly affected by this simplification.

As derived in Ref. [17, in the axially symmetric case one finds the eigenvalue equations

$$
\left(I_{1}-1\right)^{2}=I_{0} I_{2} \quad \text { or } \quad I_{1}=-1
$$

where

$$
I_{n}=\mu \int d \omega d u \frac{u^{n} g(\omega, u)}{\omega+u(\lambda+\epsilon \mu)-\Omega} .
$$

These eigenvalue equations are to be solved to find the eigenfrequencies $\Omega$ and the range of parameters where these have an imaginary part, signifying instabilities with regard to self-induced flavor conversion.

Notice that the first block in Eq. (11) provides solutions for the axially symmetric case, the so-called bimodal instability (for inverted mass ordering) and the multi-zenith-angle (MZA) instability (for normal mass ordering). The second block provides the instability where axial symmetry is spontaneously broken, the multi-azimuth-angle (MAA) instability which appears only for normal mass ordering.

\section{Switching neutrino mass ordering}

Our equations are written for inverted neutrino mass ordering (IO), whereas normal ordering (NO) corresponds to a negative $\Delta m^{2}$. However, we prefer to keep $\Delta m^{2}$ a positive parameter so that negative $\omega=\Delta m^{2} / 2 E$ continues to denote antineutrinos. We can account for the mass ordering by introducing a parameter $h= \pm 1$ in front of the vacuum oscillation term $\mathrm{M}^{2} / 2 E$ in the Hamiltonian matrix Eq. (2). This parameter carries through and finally appears in the first line of Eq. (8) in front of the term $\omega$. We can then, for NO, multiply this equation with -1 , restoring the original $\omega$ term, changing the sign of $\mu$ and $\lambda$, and the l.h.s. of the equation. For flavor oscillations, it is irrelevant if neutrinos oscillate "clockwise" or "counter-clockwise" in flavor space, i.e., the sign change on the l.h.s. is not important to find the eigenvalues. In other words, normal mass ordering is covered by

$$
\mathrm{IO} \rightarrow \mathrm{NO}: \quad \mu \rightarrow-\mu \quad \text { and } \quad \lambda \rightarrow-\lambda .
$$

Therefore, we should solve the eigenvalue equation for $-\infty<\mu, \lambda<+\infty$. The first quadrant, where both parameters are positive, corresponds to IO, the third quadrant, where both parameters are negative, to NO.

\section{E. Switching the $\nu_{e}-\bar{\nu}_{e}$ asymmetry}

We are mainly concerned with the role of the asymmetry parameter $\epsilon$. Of course, there are many ways one can modify the spectrum $g(\omega, u)$ to achieve a different asymmetry $\epsilon$. However, as a simple case we could imagine to switch the role of $\nu_{e}$ and $\bar{\nu}_{e}$, leaving all else unchanged. This modification corresponds to

$$
g(\omega, u) \rightarrow-g(-\omega, u),
$$

which implies $\epsilon \rightarrow-\epsilon$. In other words, we consider a new spectrum $\tilde{g}(\omega, u)=-g(-\omega, u)$. Following the propagation of signs in the equations, one finds that this new system is equivalent to the original one with the sign change $\lambda \rightarrow-\lambda$, whereas $\mu$ remains unchanged. More precisely, if instead of switching the sign of $\epsilon$ we switch the sign of $\lambda$, the eigenvalue will also change sign, i.e., $\Omega \rightarrow-\Omega$. Whenever $\Omega$ has an imaginary part, there exists also the 
complex conjugate solution, i.e., an exponentially growing and an exponentially damped solution. Therefore, it is irrelevant if we find $\Omega$ or $-\Omega$ because the growth rate is the same in both cases.

Finally, then, for a given spectrum $g(\omega, u)$ and studying the stability condition in the full parameter range $-\infty<\mu, \lambda<+\infty$, all four quadrants have a physical interpretation according to

$$
\begin{array}{ccc}
\multicolumn{1}{c}{} & \multicolumn{1}{c}{\mu<0} & \mu>0 \\
\lambda>0 & \text { NO, } \epsilon<0 & \text { IO, } \epsilon>0 \\
& \text { NO, } \epsilon>0 & \text { IO }, \epsilon<0 \\
& &
\end{array}
$$

where the sign change in $\epsilon$ is understood in the spirit of Eq. (14).

\section{STABILITY ANALYSIS}

\section{A. Simplified spectrum}

As a next step we perform the stability analysis for a simplified spectrum. We are not concerned with an exact numerical result for a specific SN model, but rather we wish to understand the qualitative impact of a modified $\nu_{e}-\bar{\nu}_{e}$ asymmetry when all else is kept fixed. Therefore, we construct the simplest possible toy model which allows us to develop an understanding of this question.

For the zenith-angle distribution we assume blackbody like emission from the neutrino sphere, i.e., we assume a uniform distribution of the $u$-variable on the interval $0 \leq u \leq 1$, together with a suitable reference radius $R$ as explained earlier. For the relationship between test radius $r$ and effective neutrino interaction energy $\mu$ we use the relationship shown in and around Eq. (10).

Our choices are motivated by the 3D models that have led to the discovery of the LESA phenomenon [28, 29]. Specifically, we use the model with $11.2 M_{\odot}$ progenitor mass as a benchmark for our study. During the accretion phase, this model shows large-scale convective overturn, but it does not develop the standing accretion shock instability (SASI), in contrast to the models with larger progenitor masses. After about $150 \mathrm{~ms}$ post bounce (p.b.), this and the other models develop a large-scale anisotropy of lepton-number emission.

We illustrate this behavior in Fig. 1 where we show in the top panel the flavor-dependent neutrino number fluxes in two opposite directions, roughly corresponding to the directions of maximal and minimal lepton-number flux, respectively. The $\nu_{x}$ fluxes in the two opposite directions are similar. However, in the minimum direction, the $\bar{\nu}_{e}$ flux is similar to the $\nu_{e}$ flux, corresponding to a small lepton asymmetry. In the maximum direction, the $\bar{\nu}_{e}$ and $\nu_{x}$ fluxes are similar and much smaller than the $\nu_{e}$ flux.

From these number fluxes we can derive the asymmetry parameter $\epsilon$ which we show as a function of time for
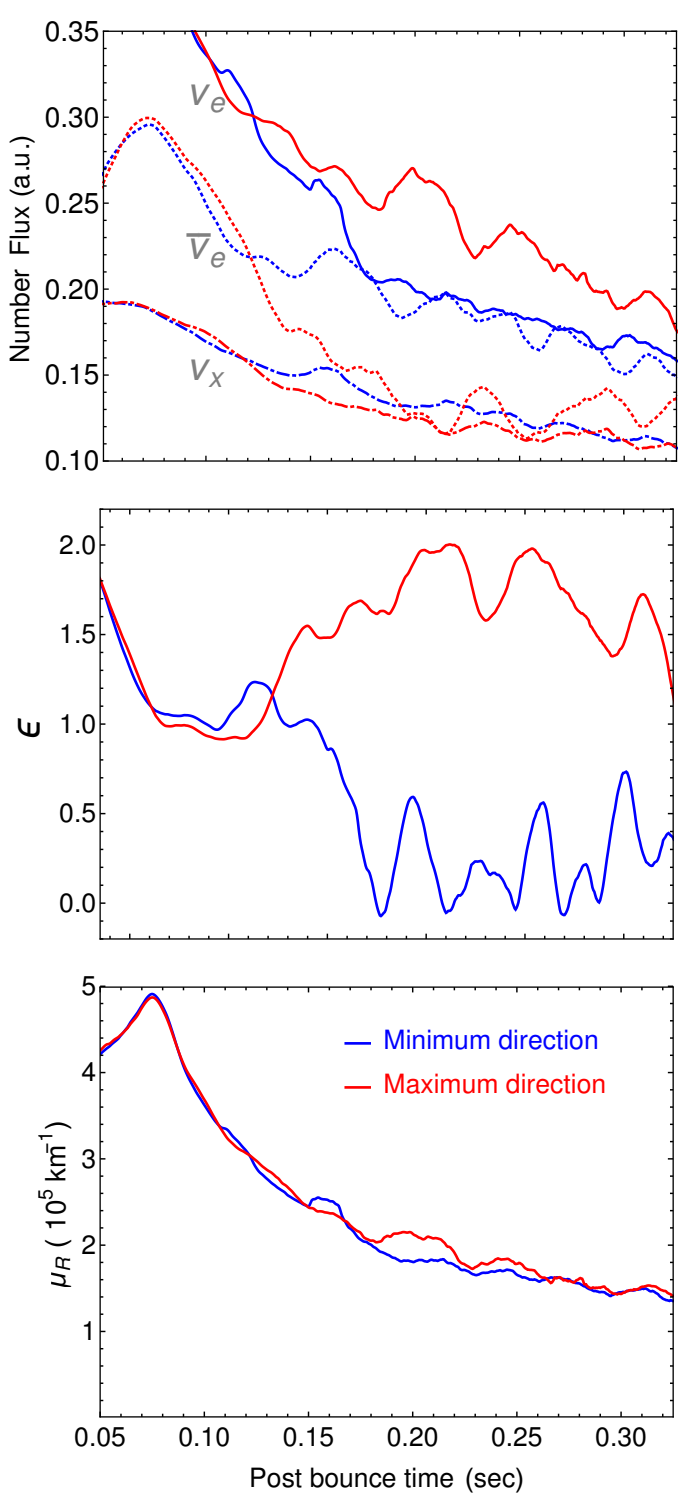

FIG. 1: Physical characteristics of our benchmark SN model in the minimum (blue lines) and maximum (red lines) leptonflux directions. The data have been smoothed (running averages) over approximately $20 \mathrm{~ms}$. Top: Number fluxes of the species $\nu_{e}, \bar{\nu}_{e}$ and $\nu_{x}$. The $\nu_{x}$ flux (representing any of $\nu_{\mu}, \bar{\nu}_{\mu}$, $\nu_{\tau}$ and $\bar{\nu}_{\tau}$ ) is similar in both directions. The $\bar{\nu}_{e}$ flux develops a large asymmetry at around $150 \mathrm{~ms}$ p.b. In the direction of minimal lepton number flux, the $\bar{\nu}_{e}$ flux is as large as the $\nu_{e}$ flux, in the opposite direction it is as small as the $\nu_{x}$ flux. Middle: Asymmetry parameter $\epsilon$ of the lepton-number flux as defined in Eq. (7). Bottom: Effective neutrino-neutrino interaction strength $\mu_{R}$ at the reference radius $R=15 \mathrm{~km}$, see Eq. 10.

our two extreme directions in the middle panel of Fig. 1. When LESA is fully developed, the $\nu_{e}$ and $\bar{\nu}_{e}$ fluxes are approximately equal in the direction of minimum leptonnumber flux so that $\epsilon \approx 0$. In the maximum lepton number flux direction, the $\bar{\nu}_{e}$ and $\bar{\nu}_{x}$ fluxes are almost exactly 
equal. In our formulation, this situation corresponds to $\epsilon=2$. In this extreme case, self-induced flavor conversion is not possible because $N_{\nu_{e}}-N_{\nu_{x}}$ cannot be swapped with $N_{\bar{\nu}_{e}}-N_{\bar{\nu}_{x}}$ if the latter is zero. Therefore, a stability analysis for $\epsilon=2$ is moot, but we will consider a case with $\epsilon=1.9$. As an intermediate benchmark case we consider a situation where $N_{\bar{\nu}_{e}}-N_{\bar{\nu}_{x}}$ is half of $N_{\nu_{e}}-N_{\nu_{x}}$, corresponding to $\epsilon=2 / 3$.

Another crucial parameter is the effective neutrinoneutrino interaction strength defined in Eq. (9a). We show $\mu_{R}$ as a function of time for our model in the bottom panel of Fig. 1. A typical value is a few $10^{5} \mathrm{~km}^{-1}$. We will specifically use the value $\mu_{R}=2 \times 10^{5} \mathrm{~km}^{-1}$ which is typical for the period after $150 \mathrm{~ms}$ when the LESA dipole is fully developed. Notice that the choice of $\mu_{R}$ is not relevant for the stability analysis per se, but only to establish a relationship between physical radius $r$ and corresponding $\mu$-value.

From past experience we know that the exact neutrino energy distribution tends to be relatively insignificant. We have explicitly checked this point by using top-hat shaped, Maxwell-Boltzmann, and more general Gamma distributions. The locus of the instability region in the $\mu-\lambda$-plane is primarily determined by the average oscillation frequency $\langle\omega\rangle$. Because we study the differential effect caused by modified $\epsilon$ values it is most transparent to use the simplest possible model which exhibits these effects. Therefore, we describe all $\nu_{e}$ and $\bar{\nu}_{e}$ by a single energy, i.e., the system is described by a vacuum oscillation frequency $\omega_{\nu}$ and one for $\omega_{\bar{\nu}}$. Moreover, we can always go to a rotating coordinate frame in flavor space such that effectively $\omega_{0} \equiv \omega_{\nu}=-\omega_{\bar{\nu}}$. Therefore, our simplified model is described by a spectrum $g(\omega, u)=h_{\epsilon}(\omega)$ which does not depend on $u$. Here,

$$
h_{\epsilon}(\omega)=\left(1+\frac{\epsilon}{2}\right) \delta\left(\omega-\omega_{0}\right)-\left(1-\frac{\epsilon}{2}\right) \delta\left(\omega+\omega_{0}\right),
$$

where the first term is for neutrinos, the second one for antineutrinos.

The abstract stability analysis does not depend on the numerical choice of $\omega_{0}$ in the sense that all other quantities of dimension "frequency," i.e., $\lambda, \mu$ and $\kappa$ are expressed in units of $\omega_{0}$. The chosen value only matters when translating the stability region in the parameter space of $\mu$ and $\lambda$ to physical SN parameters. Specifically we use $E=12 \mathrm{MeV}$ as our single energy, corresponding to $\omega_{0}=\Delta m_{\mathrm{atm}}^{2} / 2 E=0.51 \mathrm{~km}^{-1}$. Other choices would slightly shift the SN density profile relative to the instability region in our final plots Figs. 5 and 6 .

The main practical reason for using a single-energy neutrino spectrum is that it allows a simple analytic integration of the integrals Eq. (12). Avoiding a numerical integration considerably accelerates the numerical search for the eigenvalues. The imaginary part of $\Omega$, the growth rate $\kappa$, is always of order $\omega_{0}$, whereas in our range of interest, $\mu$ and $\lambda$ are up to $10^{6}$ in these units and the real part of $\Omega$ can also take on such large values. Therefore, finding the eigenvalues can be numerically challenging.

\section{B. Structure of the instability regions}

We provide the explicit eigenvalue equations for our simplified spectrum in Appendix A. For general values of $\mu, \lambda$ and $\epsilon$, the eigenvalue $\Omega$ and its imaginary part must be found numerically. The structure of typical unstable solutions is best explained using a simple example with $\epsilon=\frac{2}{3}$, corresponding to twice as many $\nu_{e}$ than $\bar{\nu}_{e}$ after subtracting the $\nu_{x}$ and $\bar{\nu}_{x}$ fluxes. We first consider the first block in Eq. (11) which derives for those solutions which preserve axial symmetry around a given radial direction. In Fig. 2 we show the growth rate $\kappa$ for unstable solutions in the parameter space consisting of the neutrino-neutrino interaction strength $\mu$ and the multiangle matter effect $\lambda$ as defined in Eq. (9). The upper right quadrant $(\mu, \lambda>0)$ of the right panel corresponds to IO and shows the traditional bimodal instability region. For a given neutrino density $\mu$, the system is stable if the matter density is either too small or too large. The same effect can be described as a shift of the unstable $\mu$ range to larger $\mu$ values for increasing $\lambda$. This effect has been termed "multi-angle matter suppression" of the instability, although the instability is not suppressed, it is shifted to larger neutrino densities. However, we can say that it is suppressed relative to a specific locus in the $\mu-\lambda$ plane which corresponds to the SN density profile.

In the lower left quadrant $(\mu, \lambda<0)$ of the right panel, corresponding with our conventions to $\mathrm{NO}$, there is no instability. Physically, of course, $\mu$ and $\lambda$ are positivechanging their sign allows us to show the NO case in the same plot as explained earlier.

When we include axial symmetry breaking a new set of solutions arises, deriving from the second block in Eq. (11), and we find the solutions shown in the left panel. This is the multi-azimuth angle (MAA) instability discussed in the previous literature. Overall, then, in IO we find the traditional bimodal instability, in NO the more recent MAA instability.

The upper left and lower right quadrants ( $\mu$ and $\lambda$ have opposite signs) are unphysical in the sense that these quadrants require an excess $\bar{\nu}_{e}$ flux. In the SN context, the collapsed material is lepton rich, implying that both the electron and neutrino densities are dominated by particles, not antiparticles, and thus that $\lambda$ and $\mu$ have the same sign. However, in the LESA context it can happen that in the direction of minimal lepton-number flux the $\bar{\nu}_{e}$ flux actually dominates. In this sense, a small negative $\epsilon$ is not entirely hypothetical, although a large negative value such as $\epsilon=-\frac{2}{3}$ would be unrealistic. Therefore, the upper left and lower right quadrants in Fig. 2 are primarily shown for mathematical completeness. In the lower right quadrant, corresponding to IO with $\epsilon=-\frac{2}{3}$, the instability range is rather insensitive to $\lambda$. In the upper left quadrant, corresponding to NO with $\epsilon=-\frac{2}{3}$, we find a total of three instabilities.

From a mathematical perspective, $\bar{\lambda}=\lambda+\epsilon \mu$ and $\mu$ is a more natural pair of parameters. Notice that in the eigenvalue equations, the matter effect enters in the form 

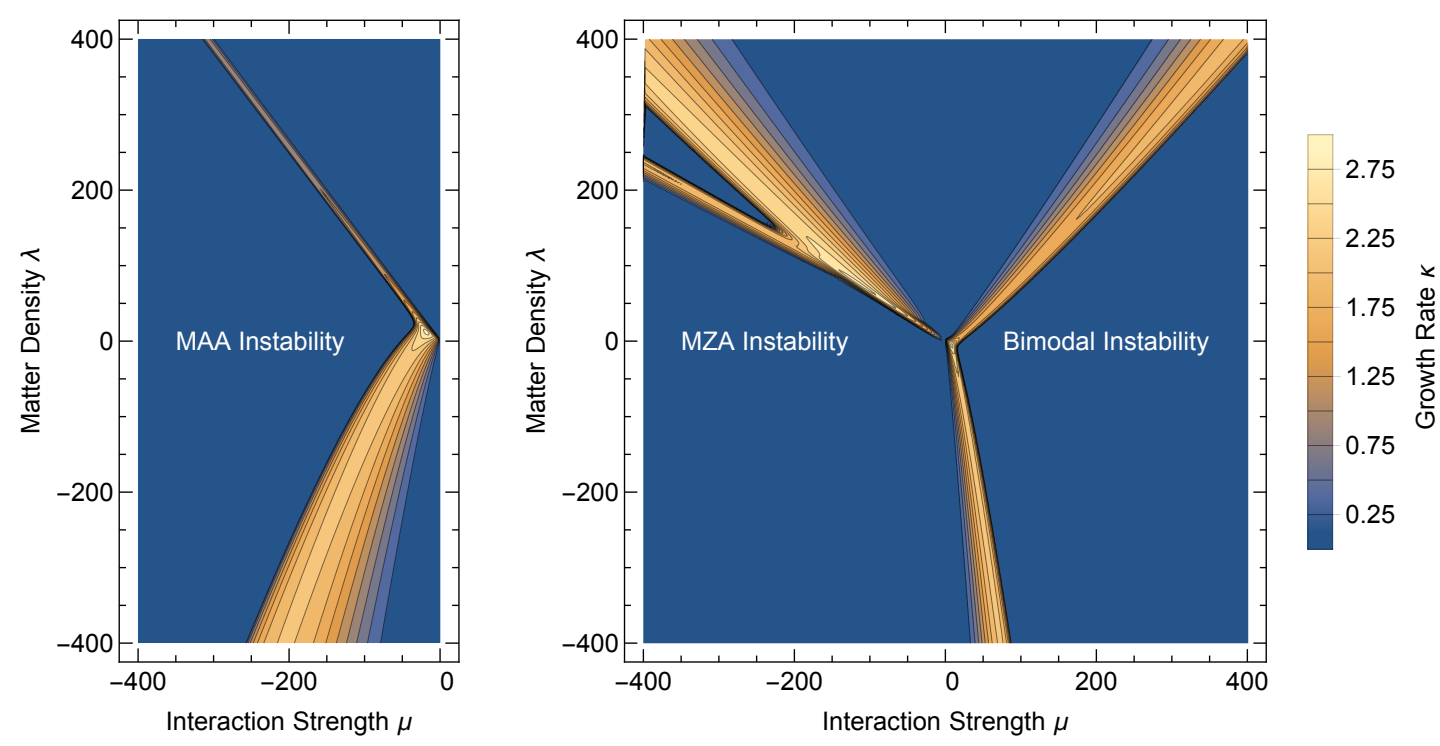

FIG. 2: Contour plot of the instability growth rate $\kappa$ for the simplified SN model described in the text. The effective neutrinoneutrino interaction strength $\mu$ and the matter effect $\lambda$ were defined in Eq. (9). Notice that $\kappa, \lambda$ and $\mu$ are given in units of the vacuum oscillation frequency $\omega_{0}$. The upper right quadrant $(\mu, \lambda>0)$ of the right panel corresponds to inverted mass ordering (IO) and the asymmetry parameter $\epsilon=+\frac{2}{3}$, shows the traditional bimodal instability. The lower left quadrant $(\mu, \lambda<0)$, corresponding to normal mass ordering (NO) and $\epsilon=+\frac{2}{3}$, shows only the MAA instability. The upper left and lower right quadrants, corresponding to opposite signs of $\mu$ and $\lambda$, represent the $\epsilon=-\frac{2}{3}$ cases. The right panel (bimodal and MZA instabilities) arises from the the first block in the eigenvalue equation (11). The left panel arises from the second block, deriving from solutions which break axial symmetry (MAA instability).
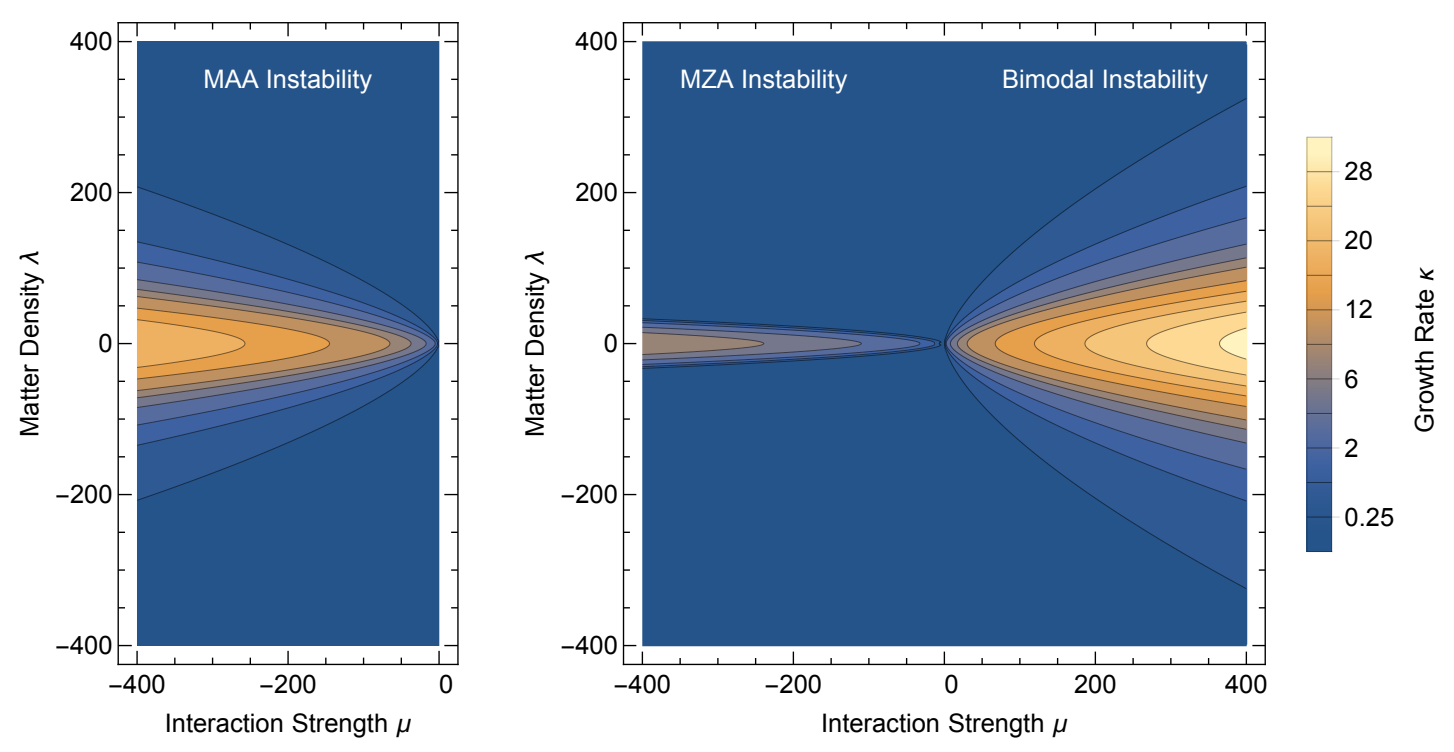

FIG. 3: Same as Fig. 2 for $\epsilon=0$. Notice the expanded scale of $\kappa$ values. In both figures, the lowest contour is for $\kappa=0.25 \omega_{0}$.

$\bar{\lambda}=\lambda+\epsilon \mu$, where $\epsilon \mu$ represents the matter effect caused by neutrinos on each other. In the $\mu-\bar{\lambda}$ parameter space, the two MZA instabilities in the right panel of Fig. 2 appear in different quadrants (cf. Fig. 9 of Ref. 27]). In other words, the line $\bar{\lambda}=\lambda+\epsilon \mu=0$, equivalent to $\lambda=-\epsilon \mu$, cuts between the two MZA instabilities.

As a second case we show in Fig. 3 a similar contour plot for the fully symmetric case $\epsilon=0$. As expected, the plot is now symmetric under the exchange $\lambda \rightarrow-\lambda$. For $\epsilon=0$ and in the absence of matter (on the line $\lambda=0$ ), the growth rate becomes arbitrarily large for $\mu \rightarrow \infty$. In this symmetric case without matter, there is no "sleeping top regime," i.e., the system is unstable for any $\mu$ above a very small threshold. Notice that the eigenvalue equation can be solved analytically for $\bar{\lambda}=0$ and any value of $\epsilon$ as shown in Appendix A. 

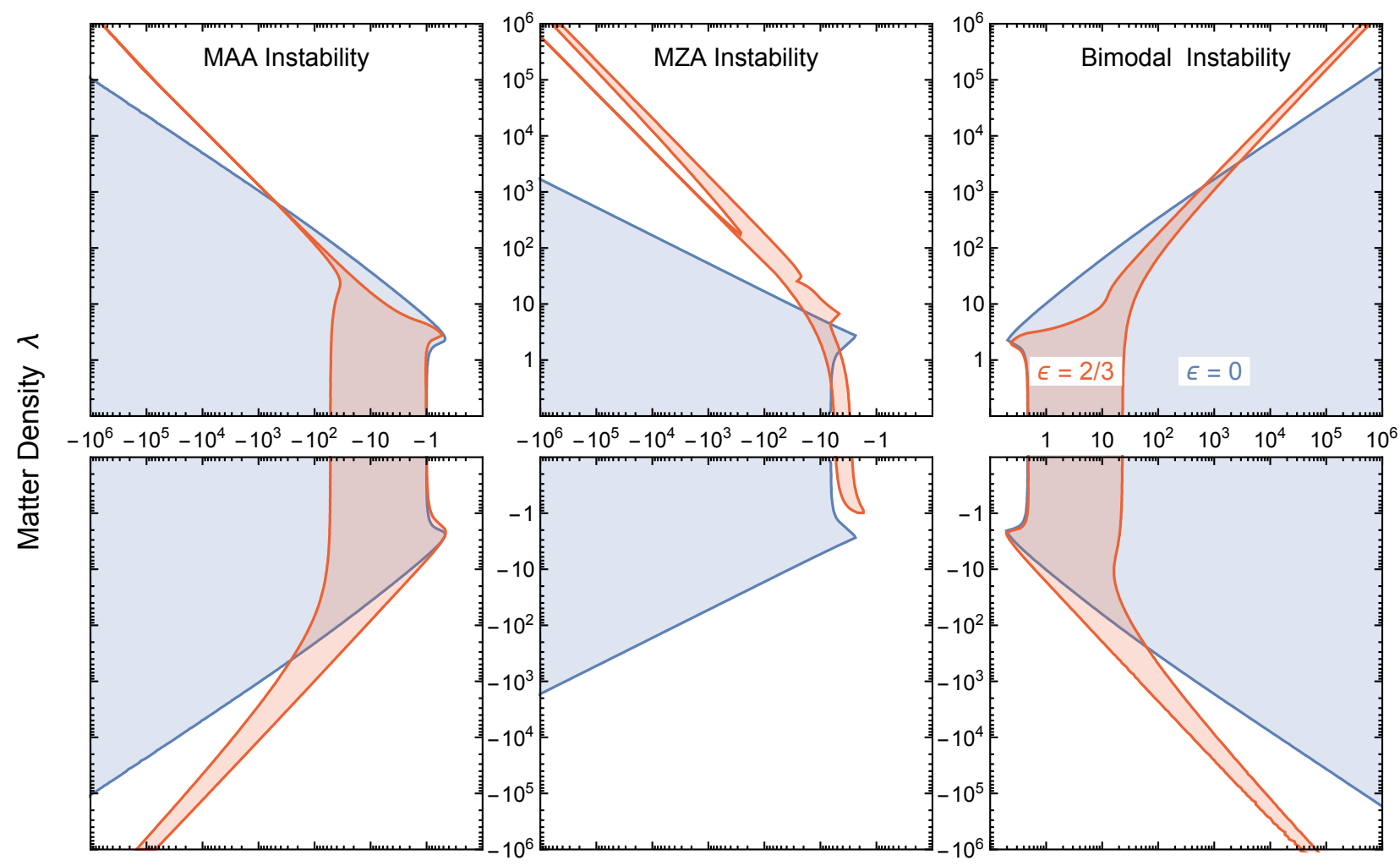

Interaction Strength $\mu$

FIG. 4: Instability footprints corresponding to the contour plots of Fig. 2 (red for $\epsilon=2 / 3$ ) and of Fig. 3 (blue for $\epsilon=0$ ), where both $\mu$ and $\lambda$ are measured in units of the vacuum oscillation frequency $\omega_{0}$. The limiting growth rate to construct the footprints is $\kappa=0.01 \omega_{0}$. For inverted mass ordering, the bimodal instability applies (upper right panel). For normal mass ordering, the lower MZA and MAA panels apply, but in practice only the lower left panel (MAA) is relevant because the MAA footprint is closer to the SN density profile than the MZA footprint.

\section{Instability footprints}

We are here not particularly interested in the details of the growth rates of the various instabilities, but rather, if neutrinos streaming from a SN core along a given density profile will encounter any instability. In this sense it is enough to find the region in the $\mu-\lambda$ parameter space where the system is unstable, a region which we call the "footprint of the instability."

This footprint is not quite uniquely defined. For example, in Fig. 2 the region to the right of the bimodal instability is perfectly stable in the sense that $\kappa=0$, whereas in the region between MZA and bimodal instability, $\kappa$ is never exactly zero but only becomes extremely small. Therefore, as a specific criteria we adopt somewhat arbitrarily $\kappa / \omega_{0}>1 / 100$ as a stability condition. The natural dimension for $\kappa$ is the vacuum oscillation frequency which in our case is of order $1 \mathrm{~km}^{-1}$. There- fore, our instability criteria corresponds to one $e$-folding of growth on a radial distance of around $100 \mathrm{~km}$. Choosing a smaller $\kappa$ criteria would imply a larger radial distance for one $e$-folding of growth. Notice also that the exact locus of the limiting $\kappa$ contour depends somewhat on the exactly chosen neutrino energy distribution which here was taken to be monochromatic. Also notice that the region nominally found unstable in this sense may not be unstable enough to lead to any appreciable growth in realistic situations. Our somewhat arbitrary stability criteria is probably conservative in this sense. In any case, we here only study the differential effect of modifying the $\epsilon$ value so that our exact choice of stability criteria is not crucial for our discussion.

In Fig. 4 we show the instability footprints of Figs. 2 and 3 , essentially providing the same information as the contour plots, but now on a logarithmic scale encompassing the range of parameters relevant in the SN context. 
For inverted mass ordering and $\epsilon \gtrsim 0$, the upper right panel (bimodal instability) applies. For large $\mu$ and large $\lambda$, the red instability range $(\epsilon=2 / 3)$ is a narrow band. As shown in Appendix $\mathrm{A}$ the asymptotic behavior of the instability strip is $\lambda \propto \mu$ except for a logarithmic correction. The curves delimiting the asymptotic footprint are provided explicitly Appendix A. For $\epsilon=0$, the asymptotic instability region is below the large- $\epsilon$ case, but in the approximate range $1 \lesssim \mu \lesssim 100$, reducing $\epsilon$ creates an instability region above the large- $\epsilon$ footprint. For the most part, however, reducing $\epsilon$ extends the unstable region to the lower right of the original footprint. We will see that this region is not of interest in the SN context.

For normal mass ordering, the panels with $\mu, \lambda<0$ apply, i.e., the lower MZA and MAA panels. The MZA footprint is covered by the MAA one, i.e., in practice the MAA instability is the only one relevant in the SN context. We see in the lower left panel that the MAA footprints are qualitatively similar to bimodal ones. Here reducing $\epsilon$ has the clear effect of shifting the instability region to an area between the red footprint and the horizontal axis, which makes it "less dangerous" in the SN context.

\section{Supernova context}

The findings of the previous section become more explicit if we focus on the physical panels of Fig. 4, i.e., the upper right and the lower left, and show the same information where $\mu$ and $\lambda$ are both plotted as positive variables and moreover, where we transform the $\mu$ parameter to radial distance in a SN model. In Figs. 5 and 6 we show the bimodal and MAA instability regions and also show a representative SN density profile inspired by our numerical LESA model. Besides the footprints already shown in Fig. 4, we now also include the case $\epsilon=1.9$ near to the largest possible value of 2 . As $\epsilon \rightarrow 2$, the footprint becomes an ever more narrow sliver of parameters. Nearmaximal $\epsilon$ values are relevant in the maximum-lepton number flux direction.

In agreement with the previous literature we find that the most "dangerous" region with regard to a possible instability is at the shock wave where the density drops by a large factor, corresponding to a much reduced multiangle matter effect. Coincidentally, in our shown example of a SN density profile, it never intersects the instability region, but of course small changes in chosen parameters or shifts in the density profile will lead to an unstable region just outside of the shock wave. For a similar result see, for example, Fig. 2 of Ref. [17.

We also confirm previous findings that the MAA instability region tends to be nearer to the SN density profile. Of course, which of the two instabilities applies depends on the neutrino mass ordering chosen by Nature. In the MAA case, reducing $\epsilon$ has the effect of shifting the unstable region away from the SN density profile. In the bimodal case, the effect is not monotonic, but typically the

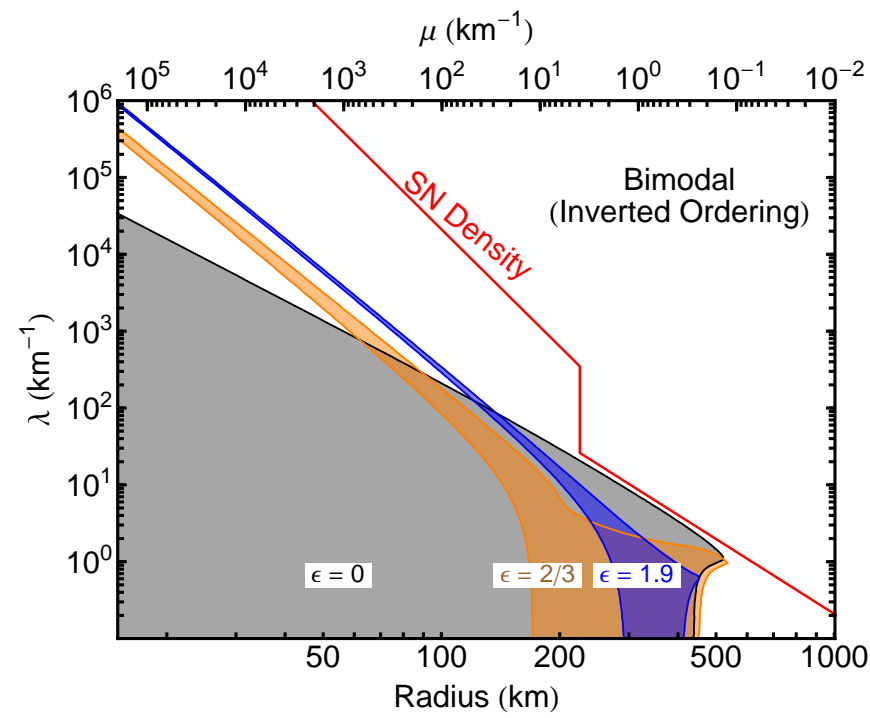

FIG. 5: Bimodal instability regions for the indicated $\epsilon$ values. The $\epsilon=2 / 3$ and 0 footprints are the same as those shown in the upper right panel of Fig. 4. We here also show the equivalent radius coordinate on the horizontal axis as well as a representative SN density profile inspired by our LESA models. The steep drop in density at around $200 \mathrm{~km}$ is the shock front.

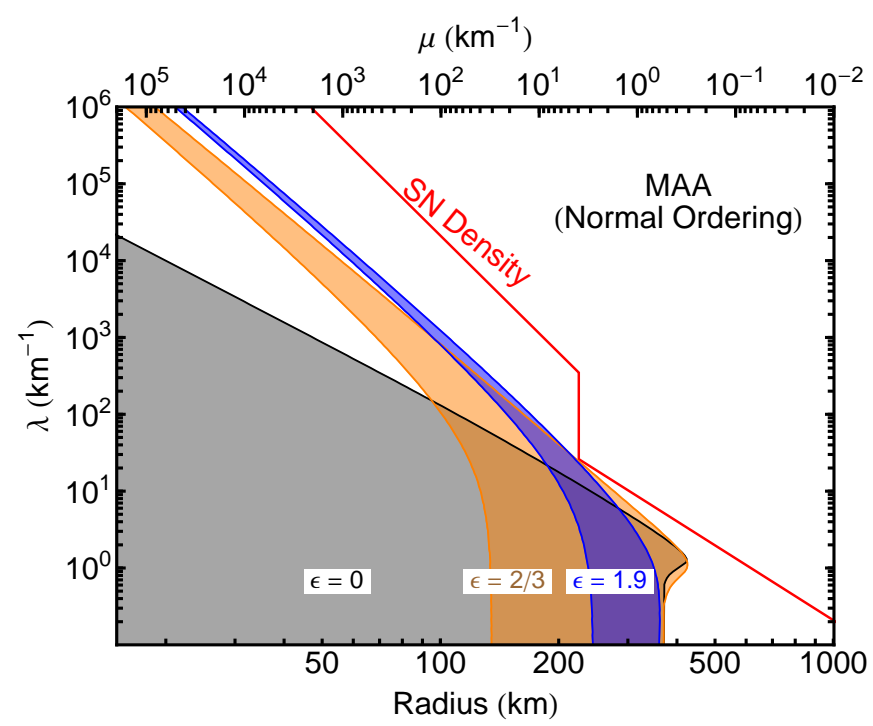

FIG. 6: Same as Fig. 5 now for the MAA instability region, corresponding to the lower left panel of Fig. 4

$\epsilon=0$ case tends to be "most dangerous" in the shockwave region and comparable to the large- $\epsilon$ case of the MAA case.

\section{CONCLUSIONS}

Motivated by the LESA effect, we have studied a simplified example of the impact of an unusually small 
lepton-number flux on the interplay between self-induced neutrino flavor conversion and its multi-angle matter suppression. The instability regions are strongly enlarged into regions of small matter density and large neutrino density as perhaps expected. However, the stability plots in Figs. 5 and 6 show that for the most part this is a region which does not correspond to a realistic SN density profile. Lowering the lepton-number flux by itself does not have the effect of leading to self-induced flavor conversion in regions below the shock front.

The main point of our study was to explore the direction of the effect of lowering the lepton-number flux. According to our simplified models even strong modifications of the asymmetry parameter do not have a dramatic impact on the stability question along realistic SN density profiles. For IO, where the bimodal instability applies, a small $\epsilon$ value can have the effect of creating an unstable region beyond the shock-wave radius. For NO, where the MAA instability applies, the largest $\epsilon$ values provide the "most dangerous" instability regions.

Overall it is primarily the multi-angle matter effect which controls the instability region for densities relevant along the SN profile. For practical SN simulations with regard to the explosion mechanism, it appears that unusually small $\epsilon$ values do not require a reconsideration of neutrino flavor evolution for distances below the shock front. Of course, flavor conversion will be important beyond of the shock front, certainly at large distances by the MSW effect, and perhaps in some cases directly outside of the shock front by self-induced flavor conversion. Such effects would be important for the interpretation of the neutrino signal from the next nearby SN, but would not directly affect the explosion dynamics.

Our stability analysis was somewhat schematic with the purpose of exploring the general direction of what an unusually small lepton-number flux caused by the LESA effect would do. In principle, of course, in any given SN simulation one could perform an a posteriori stability analysis, based on the numerical neutrino radiation field and density profile, to verify that it was justified to ignore flavor conversion. In 3D simulations, the local neutrino radiation field will not be axially symmetric, not even in the LESA dipole directions. Therefore, the stability analysis has to be based on neutrino fluxes with a nontrivial azimuth-angle distribution. This is not conceptually difficult, but such cases have not yet been explored in practice and await systematic study. In principle, one could eventually develop a numerical tool that could flag possible flavor instabilities as the numerical simulation progresses. Of course, should cases be found where flavor conversion below the shock front occurs after all, a completely new challenge has to be faced in SN modeling.

We stress, however, that not finding flavor-conversion instabilities in such an approach does not necessarily prove that none exist. As explained in the introduction, we have ignored a number of new issues that have emerged in the recent literature on neutrino-neutrino refractive effects. These topics should be sorted out before worrying further about self-induced flavor conversion in practical SN simulations. In particular, the multi-angle matter effect which provides the stabilizing ingredient in the present context relies on the assumption that the neutrino radiation field and its flavor properties vary only along the radial direction. This is an imposed symmetry assumption which can hide unstable solutions that might exist otherwise. Likewise, the assumption of a purely stationary solution, allowing us to treat the problem in the form of an ordinary (rather than partial) differential equation has never been strictly justified.

The core-collapse SN explosion mechanism is one of the few physical phenomena where neutrinos play a dominant dynamical role and where the flavor dependence of the fluxes matters. Yet flavor conversion, in spite of large mixing angles, does not seem to figure at all for SN dynamics due to matter suppression of flavor oscillations, i.e., because in dense matter, propagation and interaction eigenstates are almost the same. However, a final verdict on the role of active-active flavor conversion for SN dynamics requires more theoretical work to fully appreciate the role of possible flavor instabilities in the interacting neutrino field.

\section{Acknowledgments}

This work was partly supported by the Deutsche Forschungsgemeinschaft under Grant No. EXC-153 (Cluster of Excellence Origin and Structure of the Universe) and by the European Union under Grant No. PITN-GA-2011-289442 (FP7 Initial Training Network Invisibles). S.C. acknowledges support from the European Union through a Marie Curie Fellowship, Grant No. PIIF-GA-2011-299861. B.M. has been supported by the Australian Research Council through a Discovery Early Career Researcher Award (Grant No. DE150101145).

\section{Appendix A: Explicit eigenvalue equation}

\section{Explicit integrals}

In order to solve the eigenvalue equation we need the integrals defined in Eq. (12). In our simplified model, they take on the form

$$
I_{n}=\frac{\mu}{\bar{\lambda}} \int d \omega h_{\epsilon}(\omega) K_{n}\left(\frac{\omega-\Omega}{\bar{\lambda}}\right)
$$

where $\bar{\lambda}=\lambda+\epsilon \mu$ and

$$
K_{n}(w)=\int_{0}^{1} d u \frac{u^{n}}{u+w}
$$


and $w=(\omega-\Omega) /(\lambda+\epsilon \mu)$. We find explicitly

$$
\begin{aligned}
& K_{0}(w)=\log \left(\frac{1+w}{w}\right), \\
& K_{1}(w)=1-w \log \left(\frac{1+w}{w}\right), \\
& K_{2}(w)=\frac{1}{2}-w+w^{2} \log \left(\frac{1+w}{w}\right) .
\end{aligned}
$$

As a next step we adopt the monochromatic spectrum Eq. 16 and express all frequencies in units of $\omega_{0}$, i.e., $\mu$ is understood to mean $\mu / \omega_{0}$ and so forth. With $\bar{\lambda}=\lambda+\epsilon \mu$ we find explicitly

$$
\begin{aligned}
& I_{0}=\frac{\mu}{\bar{\lambda}}\left[\left(1+\frac{\epsilon}{2}\right) \log \left(1-\frac{\bar{\lambda}}{\Omega-1}\right)-\left(1-\frac{\epsilon}{2}\right) \log \left(1-\frac{\bar{\lambda}}{\Omega+1}\right)\right], \\
& I_{1}=\frac{\mu}{\bar{\lambda}^{2}}\left[\epsilon \bar{\lambda}+\left(1+\frac{\epsilon}{2}\right)(\Omega-1) \log \left(1-\frac{\bar{\lambda}}{\Omega-1}\right)-\left(1-\frac{\epsilon}{2}\right)(\Omega+1) \log \left(1-\frac{\bar{\lambda}}{\Omega+1}\right)\right], \\
& I_{2}=\frac{\mu}{\bar{\lambda}^{3}}\left[\bar{\lambda}\left(\frac{\epsilon \bar{\lambda}}{2}+\epsilon \Omega-2\right)+\left(1+\frac{\epsilon}{2}\right)(\Omega-1)^{2} \log \left(1-\frac{\bar{\lambda}}{\Omega-1}\right)-\left(1-\frac{\epsilon}{2}\right)(\Omega+1)^{2} \log \left(1-\frac{\bar{\lambda}}{\Omega+1}\right)\right] .
\end{aligned}
$$

\section{Vanishing effective matter density $(\bar{\lambda} \rightarrow 0)$}

In the absence of matter effects, where $\bar{\lambda}=\lambda+\epsilon \mu \rightarrow 0$, these expressions simplify to

$$
I_{0}=\mu \frac{2+\epsilon \Omega}{1-\Omega^{2}}, \quad I_{1}=\frac{1}{2} I_{0}, \quad \text { and } \quad I_{2}=\frac{1}{3} I_{0} .
$$

In this case the eigenvalue equations can be solved explicitly,

$$
\begin{aligned}
\Omega_{\text {bimodal }} & =-\frac{2 \sqrt{3}+3}{12} \epsilon \mu \\
& \pm \sqrt{1-\frac{2 \sqrt{3}+3}{3} \mu+\frac{7+4 \sqrt{3}}{48}(\epsilon \mu)^{2}} \\
\Omega_{\mathrm{MZA}} & =+\frac{2 \sqrt{3}-3}{12} \epsilon \mu \\
& \pm \sqrt{1+\frac{2 \sqrt{3}-3}{3} \mu+\frac{7-4 \sqrt{3}}{48}(\epsilon \mu)^{2}} \\
\Omega_{\mathrm{MAA}} & =\frac{\epsilon \mu}{4} \pm \sqrt{1+\mu+\left(\frac{\epsilon \mu}{4}\right)^{2}} .
\end{aligned}
$$

For these solutions to have a nonvanishing imaginary part, the bimodal solution requires $\mu>0$, whereas the two others require $\mu<0$. For all three cases, one finds that the maximum growth rate to be

$$
\kappa_{\max }=\sqrt{(2 / \epsilon)^{2}-1},
$$

which of course only applies on the locus $\bar{\lambda}=0$, i.e., on the line $\lambda=-\epsilon \mu$. In other regions of the $\mu-\lambda$ parameter space, $\kappa_{\max }$ could be larger. Notice that in our convention, $-2 \leq \epsilon \leq+2$ so that $\kappa_{\max }$ is indeed real and positive. For small $\epsilon$, we have $\kappa_{\max } \approx|2 / \epsilon|$. Recalling that $\kappa$ is given in units of the vacuum oscillation frequency $\omega_{0}$ we find once more that the maximum growth rate is essentially identical with the vacuum oscillation frequency times a numerical factor depending on $\epsilon$.

The situation changes in the absence of any $\nu_{e}-\bar{\nu}_{e}$ asymmetry where $\epsilon=0$. In this case the imaginary parts of all three solutions grow without limit for $|\mu| \rightarrow \infty$. For $|\mu|=400$, the edge of the plotting region in Fig. 3 and for $\lambda=0$ we find $\kappa_{\text {bimodal }}=29.34, \kappa_{\mathrm{MZA}}=7.93$, and $\kappa_{\mathrm{MAA}}=20.02$, in agreement with the contours in Fig. 3 .

\section{Large effective matter density $(\bar{\lambda} \rightarrow \pm \infty)$}

In the opposite limit of very large effective matter density $\bar{\lambda} \rightarrow \infty$ we may also gain considerable insight by analytic techniques. To this end we observe that unstable solutions, for large $\lambda$ values, also require large $\mu$ values. Therefore, to expand in powers of $1 / \bar{\lambda}$, we express $\mu=m \bar{\lambda}$, where $m$ is a dimensionless parameter. Of course, at this stage this is only an ansatz because $\mu$ could also scale with another power of $\bar{\lambda}$.

Considering first $\bar{\lambda}>0$ we assume that the real part of $\Omega$ does not become large, i.e., that $|\Omega|$ can be considered small relative to $\bar{\lambda}$ or $\mu$. Under these conditions, to lowest order in $1 / \bar{\lambda}$ the first block of the eigenvalue equation 11 becomes

$$
\frac{(2+\epsilon) \log (1-\Omega)-(2-\epsilon) \log (-1-\Omega)}{2 \epsilon}=a,
$$

where

$$
a=\log (\bar{\lambda})-\frac{2(m-1)^{2}}{m^{2}} .
$$

The second block, yielding the MAA instability, provides 
to lowest order

$$
m=-1 \text {. }
$$

Therefore, to lowest order in $1 / \bar{\lambda}$, any solution, stable or unstable, requires $\mu=-\bar{\lambda}$. Therefore, with $\bar{\lambda}=\lambda+\epsilon \mu$ we find

$$
\mu=-\frac{\lambda}{1+\epsilon} .
$$

This results corresponds to the very thin footprint in the upper left panel of Fig. 4 .

Next we turn to $\bar{\lambda} \rightarrow-\infty$. We notice without further discussion that in this case the real part of $\Omega$ becomes of order $\bar{\lambda}$ so that we write $\Omega \rightarrow \bar{\lambda}+\Omega$. Performing now the expansion, we find for both blocks of Eq. (11) a result of the form of Eq. A8, but with modified expressions

$$
\begin{aligned}
& a=\log (-\bar{\lambda})-\frac{2(m-1)^{2}}{m(m-4)}, \\
& a=\log (-\bar{\lambda})-\frac{m+1}{m},
\end{aligned}
$$

which appear on the right-hand side.

Equation A8 can be solved explicitly for our benchmark example $\epsilon=2 / 3$ where it is essentially a quadratic equation. In this case one finds

$$
\Omega=\frac{2-e^{a} \pm e^{a / 2} \sqrt{e^{a}-8}}{2}
$$

This solution has a nonvanishing imaginary part for $-\infty<a<\log (8)$. However, for $a \ll-1$ the imaginary part (the growth rate) is exponentially suppressed.
The formal criteria $\kappa>1 / 100$ leads to the requirement $a>\log (4-\sqrt{39999} / 50)$. Therefore, the system is unstable for $a_{1}<a<a_{2}$, where

$$
\begin{aligned}
& a_{1}=\log (4-\sqrt{39999} / 50) \approx-9.90, \\
& a_{2}=\log (8) \approx+2.08,
\end{aligned}
$$

assuming our nominal requirement $\kappa>1 / 100$.

To make these results more explicit for the various instabilities, we first solve Eq. A9, corresponding to $\bar{\lambda} \rightarrow+\infty$. We find

$$
\mu=\frac{\bar{\lambda}}{1 \pm \sqrt{(\log \bar{\lambda}-a) / 2}}
$$

Together with $\lambda=\bar{\lambda}-\epsilon \mu$ and with the explicit values for $a_{1}$ and $a_{2}$ we can find the limiting contours in the $\mu$ - $\lambda$ plane as a parametric plot depending on the variable $\bar{\lambda}$.

Likewise, we may solve Eq. A12, corresponding to $\bar{\lambda} \rightarrow-\infty$. Here we find

$$
\begin{aligned}
\mu & =+\frac{\bar{\lambda}}{1+A \pm \sqrt{\frac{3}{2} A+A^{2}}}, \\
\mu & =-\frac{\bar{\lambda}}{1+A},
\end{aligned}
$$

where

$$
A=a-\log \bar{\lambda}
$$

Again we can find the limiting contours with $a_{1}$ and $a_{2}$ in the $\mu$ - $\lambda$-plane as a parametric plot depending on the variable $\bar{\lambda}$.
[1] A. Mirizzi, I. Tamborra, H.-T. Janka, N. Saviano, K. Scholberg, R. Bollig, L. Hüdepohl and S. Chakraborty, "Supernova neutrinos: Production, oscillations and detection," arXiv:1508.00785.

[2] L. Wolfenstein, "Neutrino oscillations in matter," Phys. Rev. D 17, 2369 (1978).

[3] S. P. Mikheev and A. Yu. Smirnov, "Resonance enhancement of oscillations in matter and solar neutrino spectroscopy," Sov. J. Nucl. Phys. 42, 913 (1985) [Yad. Fiz. 42, 1441 (1985)]; "Neutrino oscillations in a variable density medium and neutrino bursts due to the gravitational collapse of stars," Sov. Phys. JETP 64, 4 (1986) [Zh. Eksp. Teor. Fiz. 91, 7 (1986)].

[4] A. S. Dighe and A. Yu. Smirnov, "Identifying the neutrino mass spectrum from the neutrino burst from a supernova," Phys. Rev. D 62, 033007 (2000) hep$\mathrm{ph} / 9907423$.

[5] J. Pantaleone, "Neutrino oscillations at high densities," Phys. Lett. B 287, 128 (1992).

[6] V. A. Kostelecký and S. Samuel, "Neutrino oscillations in the early universe with an inverted neutrino mass hierarchy," Phys. Lett. B 318, 127 (1993).
[7] S. Samuel, "Bimodal coherence in dense selfinteracting neutrino gases," Phys. Rev. D 53, 5382 (1996) hep-ph/ 9604341].

[8] H. Duan, G. M. Fuller and Y.-Z. Qian, "Collective neutrino flavor transformation in supernovae," Phys. Rev. D 74, 123004 (2006) astro-ph/0511275.

[9] H. Duan, G. M. Fuller, J. Carlson and Y.-Z. Qian, "Simulation of coherent non-linear neutrino flavor transformation in the supernova environment: Correlated neutrino trajectories," Phys. Rev. D 74, 105014 (2006) astro$\mathrm{ph} / 0606616$.

[10] H. Duan, G. M. Fuller and Y.-Z. Qian, "Collective neutrino oscillations," Annu. Rev. Nucl. Part. Sci. 60, 569 (2010) arXiv:1001.2799.

[11] A. Banerjee, A. Dighe and G. Raffelt, "Linearized flavorstability analysis of dense neutrino streams," Phys. Rev. D 84, 053013 (2011) arXiv:1107.2308.

[12] A. Esteban-Pretel, A. Mirizzi, S. Pastor, R. Tomàs, G. G. Raffelt, P. D. Serpico and G. Sigl, "Role of dense matter in collective supernova neutrino transformations," Phys. Rev. D 78, 085012 (2008) arXiv:0807.0659.

[13] S. Chakraborty, T. Fischer, A. Mirizzi, N. Saviano and 
R. Tomàs, "No collective neutrino flavor conversions during the supernova accretion phase," Phys. Rev. Lett. 107, 151101 (2011) arXiv:1104.4031.

[14] S. Chakraborty, T. Fischer, A. Mirizzi, N. Saviano and R. Tomàs, "Analysis of matter suppression in collective neutrino oscillations during the supernova accretion phase," Phys. Rev. D 84, 025002 (2011) arXiv:1105.1130.

[15] B. Dasgupta, E. P. O'Connor and C. D. Ott, "The role of collective neutrino flavor oscillations in core-collapse supernova shock revival,” Phys. Rev. D 85, 065008 (2012) arXiv:1106.1167.

[16] S. Sarikas, G. G. Raffelt, L. Hüdepohl and H.-T. Janka, "Suppression of self-induced flavor conversion in the supernova accretion phase," Phys. Rev. Lett. 108, 061101 (2012) arXiv:1109.3601.

[17] G. Raffelt, S. Sarikas and D. de Sousa Seixas, "Axial symmetry breaking in self-induced flavor conversion of supernova neutrino fluxes," Phys. Rev. Lett. 111, 091101 (2013) arXiv:1305.7140.

[18] G. Raffelt and D. de Sousa Seixas, "Neutrino flavor pendulum in both mass hierarchies," Phys. Rev. D 88, 045031 (2013) arXiv:1307.7625.

[19] A. Mirizzi, "Multi-azimuthal-angle effects in self-induced supernova neutrino flavor conversions without axial symmetry," Phys. Rev. D 88, 073004 (2013) arXiv:1308.1402.

[20] S. Chakraborty and A. Mirizzi, "Multi-azimuthal-angle instability for different supernova neutrino fluxes," Phys. Rev. D 90, 033004 (2014) arXiv:1308.5255.

[21] R. S. Hansen and S. Hannestad, "Chaotic flavor evolution in an interacting neutrino gas," Phys. Rev. D 90, 025009 (2014) arXiv:1404.3833.

[22] S. Chakraborty, A. Mirizzi, N. Saviano and D. de Sousa Seixas, "Suppression of the multi-azimuthalangle instability in dense neutrino gas during supernova accretion phase," Phys. Rev. D 89, 093001 (2014) arXiv:1402.1767.

[23] G. Mangano, A. Mirizzi and N. Saviano, "Damping the neutrino flavor pendulum by breaking homogeneity," Phys. Rev. D 89, 073017 (2014) arXiv:1403.1892.

[24] H. Duan and S. Shalgar, "Flavor instabilities in the neutrino line model," Phys. Lett. B 747, 139 (2015) arXiv:1412.7097.

[25] A. Mirizzi, G. Mangano and N. Saviano, "Self-induced flavor instabilities of a dense neutrino stream in a twodimensional model," Phys. Rev. D 92, 021702 (2015) arXiv:1503.03485.

[26] A. Mirizzi, "Breaking the symmetries of the bulb model in two-dimensional self-induced supernova neutrino flavor conversions," arXiv:1506.06805.

[27] S. Chakraborty, R. S. Hansen, I. Izaguirre and G. Raffelt, "Self-induced flavor conversion of supernova neutrinos on small scales," arXiv:1507.07569.

[28] I. Tamborra, F. Hanke, H.-T. Janka, B. Müller, G. G. Raffelt and A. Marek, "Self-sustained asymmetry of lepton-number emission: A new phenomenon during the supernova shock-accretion phase in three dimensions," Astrophys. J. 792, 96 (2014) arXiv:1402.5418.

[29] I. Tamborra, G. Raffelt, F. Hanke, H.-T. Janka and B. Müller, "Neutrino emission characteristics and detection opportunities based on three-dimensional supernova simulations," Phys. Rev. D 90, 045032 (2014) arXiv:1406.0006.
[30] T. Melson, H.-T. Janka and A. Marek, "Neutrino-driven supernova of a low-mass iron-core progenitor boosted by three-dimensional turbulent convection," Astrophys. J. 801, L24 (2015) arXiv:1501.01961.

[31] T. Melson, H.-T. Janka, R. Bollig, F. Hanke, A. Marek and B. Müller, "Neutrino-driven explosion of a 20 solarmass star in three dimensions enabled by strange-quark contributions to neutrino-nucleon scattering," Astrophys. J. 808, L42 (2015) arXiv:1504.07631.

[32] E. J. Lentz et al., "Three-dimensional core-collapse supernova simulated using a $15 M_{\odot}$ progenitor," Astrophys. J. 807, L31 (2015) arXiv:1505.05110.

[33] H.-T. Janka, "Three-dimensional core-collapse simulations by the Garching Group: The route to explosions," Talk at F.O.E. Fifty-One Erg, Raleigh, NC, 1-5 June 15, http://www.physics.ncsu.edu/FOE2015 /PRESENTATIONS/Janka_Raleigh-2015.pdf

[34] Eric Lentz, private communication (2015).

[35] E. Müller, H.-T. Janka and A. Wongwathanarat, "Parametrized 3D models of neutrino-driven supernova explosions: Neutrino emission asymmetries and gravitationalwave signals," Astron. Astrophys. 537, 63 (2012) arXiv:1106.6301.

[36] S. Hannestad, G. G. Raffelt, G. Sigl and Y. Y. Y. Wong, "Self-induced conversion in dense neutrino gases: Pendulum in flavour space," Phys. Rev. D 74, 105010 (2006); Erratum ibid. 76, 029901 (2007) astro-ph/0608695.

[37] H. Duan, G. M. Fuller, J. Carlson and Y.-Z. Qian, "Analysis of collective neutrino flavor transformation in supernovae," Phys. Rev. D 75, 125005 (2007) astro$\mathrm{ph} / 0703776$.

[38] G. G. Raffelt and G. Sigl, "Self-induced decoherence in dense neutrino gases," Phys. Rev. D 75, 083002 (2007) hep-ph/0701182.

[39] A. Esteban-Pretel, S. Pastor, R. Tomàs, G. G. Raffelt and G. Sigl, "Decoherence in supernova neutrino transformations suppressed by eleptonization," Phys. Rev. D 76, 125018 (2007) arXiv:0706.2498.

[40] C. Giunti and A. Studenikin, "Neutrino electromagnetic interactions: A window to new physics," Rev. Mod. Phys. 87 (2015) 531 arXiv:1403.6344.

[41] A. de Gouvea and S. Shalgar, "Effect of transition magnetic moments on collective supernova neutrino oscillations," JCAP 1210, 027 (2012) arXiv:1207.0516.

[42] A. de Gouvea and S. Shalgar, "Transition magnetic moments and collective neutrino oscillations: Threeflavor effects and detectability," JCAP 1304, 018 (2013) arXiv:1301.5637.

[43] A. I. Studenikin, "Neutrinos in electromagnetic fields and moving media," Phys. Atom. Nucl. 67 (2004) 993 [Yad. Fiz. 67 (2004) 1014].

[44] A. Vlasenko, G. M. Fuller and V. Cirigliano, "Neutrino quantum kinetics," Phys. Rev. D 89, 105004 (2014) arXiv:1309.2628.

[45] V. Cirigliano, G. M. Fuller and A. Vlasenko, "A new spin on neutrino quantum kinetics," Phys. Lett. B 747, 27 (2015) arXiv:1406.5558.

[46] A. Vlasenko, G. M. Fuller and V. Cirigliano, "Prospects for neutrino-antineutrino transformation in astrophysical environments," arXiv:1406.6724.

[47] C. Volpe, D. Väänänen and C. Espinoza, "Extended evolution equations for neutrino propagation in astrophysical and cosmological environments," Phys. Rev. D 87, 113010 (2013) arXiv:1302.2374. 
[48] D. Väänänen and C. Volpe, "Linearizing neutrino evolution equations including neutrino-antineutrino pairing correlations," Phys. Rev. D 88, 065003 (2013) arXiv:1306.6372.

[49] J. Serreau and C. Volpe, "Neutrino-antineutrino correlations in dense anisotropic media," Phys. Rev. D 90, 125040 (2014) arXiv:1409.3591.

[50] A. Kartavtsev, G. Raffelt and H. Vogel, "Neutrino propagation in media: Flavor, helicity, and pair correlations," Phys. Rev. D 91, 125020 (2015) arXiv:1504.03230.

[51] J. F. Cherry, J. Carlson, A. Friedland, G. M. Fuller and A. Vlasenko, "Neutrino scattering and flavor transformation in supernovae," Phys. Rev. Lett. 108, 261104 (2012) [arXiv: 1203.1607].

[52] J. F. Cherry, J. Carlson, A. Friedland, G. M. Fuller and A. Vlasenko, "Halo modification of a supernova neutronization neutrino burst," Phys. Rev. D 87, 085037 (2013) arXiv:1302.1159.

[53] S. Sarikas, I. Tamborra, G. Raffelt, L. Hüdepohl and H.-T. Janka, "Supernova neutrino halo and the suppres- sion of self-induced flavor conversion," Phys. Rev. D 85 113007 (2012) arXiv:1204.0971.

[54] A. Mirizzi and P. D. Serpico, "Flavor stability analysis of dense supernova neutrinos with flavor-dependent angular distributions," Phys. Rev. D 86, 085010 (2012) arXiv:1208.0157.

[55] A. Marek and H.-T. Janka, "Delayed neutrino-driven supernova explosions aided by the standing accretionshock instability," Astrophys. J. 694, 664 (2009) arXiv:0708.3372].

[56] B. Müller, H.-T. Janka and A. Marek, "A new multidimensional general relativistic neutrino hydrodynamics code of core-collapse supernovae III. Gravitational wave signals from supernova explosion models," Astrophys. J. 766, 43 (2013) arXiv:1210.6984.

[57] H.-T. Janka, F. Hanke, L. Hüdepohl, A. Marek, B. Müller and M. Obergaulinger, "Core-collapse supernovae: Reflections and directions," PTEP 2012, 01A309 (2012) arXiv:1211.1378. 